\title{
Lafora Disease
}

National Cancer Institute

\section{Source}

National Cancer Institute. Lafora Disease. NCI Thesaurus. Code C84804.

A rare, fatal autosomal recessive inherited disorder caused by mutations in the genes EPM2A and EPM2b. It is characterized by the presence of cytoplasmic inclusion bodies called Lafora bodies in many cells of the body including neurons, muscle cells, and liver cells. The Lafora bodies contain mucopolysaccharides. Signs and symptoms include seizures, myoclonus, ataxia, and dementia. 